\title{
Parents of young people with mental health problems experienced a deskilling and had to learn to reskill themselves
}

Harden J. "Uncharted waters": the experience of parents of young people with mental health problems. Qual Health Res 2005; 15:207-23.

\section{Q What are the experiences of parents who care for a young person with mental health problems?}

\section{DESIGN}

Qualitative study using semistructured, indepth interviews.

\section{SETTING}

UK.

\section{PARTICIPANTS}

25 parents ( 18 mothers, 7 fathers) of 18 young people (13-16 y of age) with mental health problems. All families were white and had various socioeconomic backgrounds.

\section{METHODS}

Parents were interviewed about the following topics: the nature of their child's problem; relationships with health professionals; implications for their lifestyle, health, employment, and education; impact on the family; and coping. Interviews were audiotaped, transcribed, and analysed qualitatively using an interpretive inductive approach.

\section{MAIN FINDINGS}

Parental deskilling: (1) Not being listened to by the medical profession. Parents felt their role was challenged because they were unfamiliar with mental health problems and had to rely on experts for explanations. When they sought help to overcome their inexperience, it was not always forthcoming, which led to feelings of isolation, abandonment, and self doubt. Their confidence was undermined when they were accused of overreacting to what was considered normal adolescent changes. (2) Helpless parenting. Parents had to negotiate with health professionals in initial medical encounters, which was often distressing. They felt helpless and frustrated by the lack of progress. The information they received was considered a useful start but inadequate for the practicalities of continuing to care for their children. (3) Being excluded from care. Health professionals took over some of the caring responsibilities that were previously done by parents, which left them feeling isolated. When young people were $\geqslant 16$ years of age, parents' roles were further limited, and they were formally excluded from decision making. Parents were frustrated because their children's mental health problems meant that their children did not recognise when they needed help. Parents' concerns were exacerbated when children were admitted to hospital, especially if they were admitted to adult wards. (4) Parental causal responsibility. Parents often felt responsible for their children's conditions. Their parental skills came under self scrutiny as well as scrutiny by the medical profession. Guilt was expressed in the early stages of the illness. Parents often felt judged by health professionals, and they questioned their own ability to parent.

Parental reskilling: (1) Critiquing psychiatric knowledge and practice. Although parents sought professional help, they were critical of the medical profession and challenged the expert status of psychiatric knowledge. They critiqued the difficulty of getting a clear diagnosis and effective treatment for their children. Their helplessness led them to acquire knowledge and to begin to reskill themselves. (2) Acquiring knowledge. Parents independently sought information to supplement the information given by healthcare professionals. They became more aware of information about the illness. Although parents felt able to

For correspondence: Dr $\mathrm{j}$ Harden, Napier University, Edinburgh, UK. i.harden@napier.ac.uk

Source of funding: not stated. challenge health professionals over general issues, they deemed that specific matters (eg, medication) were beyond their area of knowledge. (3) Renegotiating the parental caregiving role. Parents found ways to accept and integrate their children's conditions in their daily lives. Sometimes, though, they used phrases such as "get on with it" to fill in a gap or to minimise the emotional impact of their narrative. Parents also renegotiated care roles with their children and health professionals so that they could maintain some control and have access to information about their children's care.

\section{CONCLUSIONS}

Parents of young people with mental health problems found that their caregiving role changed through interactions with health professionals. Their lack of knowledge and inexperience left them feeling isolated and helpless. By acquiring knowledge and renegotiating the parental caregiving role, parents were able to reskill themselves.

\section{Commentary}

he qualitative study by Harden builds on earlier evidence of the longstanding communication difficulties between professionals and family caregivers during periods of intense need or crisis. ${ }^{1}$ Mental illness, often chronic and long term, profoundly affects both the individual with the illness and family members, who must cope with feelings of uncertainty, confusion, devastating loss, and helplessness.

These insights into the emotional reactions and perceptions of parents help healthcare professionals to empathise with parents. The study highlights the conflict that can occur between parental and professional explanations of mental health problems. Harden identified the parental desire to create a role for themselves on the basis of their own knowledge and skills. Despite the pivotal roles of families as caregivers in facilitating recovery, family needs are frequently overlooked or minimised, with parents sometimes feeling blamed for their children's ill health. Parents may interpret nurses' attitudes as unprofessional and uncaring, resulting in greater anguish. Incorporating family interventions can prevent relapse, improve health outcomes, and reduce overall healthcare costs. ${ }^{2}$ Barriers to family centred practice include clinicians' lack of empathy, lack of understanding of family member needs, and absent skills in assessing, intervening, and supporting families, as well as the frequently cited "time crunch." Family centred practice could be promoted by developing practitioners' skills in therapeutic communication, empathy training, and active listening. Managing the tensions that arise when balancing the need to focus care on young people and simultaneously involve their families generates some difficult decisions. The study by Harden suggests that recognising that parents have knowledge and skills that they wish health professionals to value may contribute to more effective family centred care and client outcomes. Greater opportunities for development of therapeutic communication skills are needed to enhance nursing involvement with patients.

\section{Scheannette LeGris, RN, MHSc Jeannette LeGris, RN, MHSc
Nursing, McMaster University Hamilton, Ontario, Canada}

1 Wright LM, Leahey M. Maximizing time, minimizing suffering: the 15minute (or less) family interview. Journal of Family Nursing 1999;5:25974.

2 Lehman AF, Steinwachs DM. Patterns of usual care for schizophrenia: initial results from the Schizophrenia Patient Outcomes Research Team (PORT) Client Survey. Schizophr Bull 1998;24:11-20.

3 Wright LM, Leahey M. Nurses and families: a guide to assessment and intervention. Philadelphia: FA Davis, 2000. 